A N N A L E S Annales de Bretagne et des Pays de l'Ouest

\title{
Les parlements de l'Ouest : archives, logiques et pratiques institutionnelles
}

David Feutry et Gauthier Aubert

\section{OpenEdition}

\section{Journals}

Édition électronique

URL : http://journals.openedition.org/abpo/3104

DOI : $10.4000 / a b p o .3104$

ISSN : 2108-6443

Éditeur

Presses universitaires de Rennes

Édition imprimée

Date de publication : 31 octobre 2015

Pagination : 7-9

ISSN : 0399-0826

\section{Référence électronique}

David Feutry et Gauthier Aubert, « Les parlements de l'Ouest : archives, logiques et pratiques

institutionnelles », Annales de Bretagne et des Pays de l'Ouest [En ligne], 122-3 | 2015, mis en ligne le 30 octobre 2015, consulté le 24 avril 2019. URL : http://journals.openedition.org/abpo/3104 


\title{
Introduction \\ Les parlements de l'Ouest : archives, logiques et pratiques institutionnelles
}

\author{
David FEUTRY \\ Docteur en histoire moderne - université Paris-Sorbonne
}

Gauthier AUBERT

Maître de conférences en histoire moderne

- CERHIO UMR 6258, université Rennes 2

\begin{abstract}
«Richesse et fonctions font de cette caste de plus en plus fermée, fière et gourmée, un instrument de réaction nobiliaire et seigneuriale, bien marquée par l'esprit de ses arrêts de justice [...]. La magistrature se dresse contre le courant du siècle, contre les réformes institutionnelles, contre la philosophie, dont elle fait brûler quelques ouvrages impies, contre les protestants enfin, combats d'arrière-garde où, dans quelques procès retentissants, le fanatisme n'est pas du clergé, mais bien de la robe ${ }^{1}$. "
\end{abstract}

Hubert Méthivier résumait parfaitement ainsi, il y a cinquante ans, l'avis partagé par de nombreux historiens sur les parlements d'Ancien Régime. Peuplés de dynasties de privilégiés, bourreaux de Calas autant que des philosophes, ils jouèrent un rôle indéniable de trublion lors des crises politiques de la monarchie, depuis la Ligue jusqu'à la Fronde. Malgré une soumission éphémère sous Louis XIV, ils n'hésitèrent pas à contester les réformes monarchiques durant tout le $\mathrm{XVIII}^{\mathrm{e}}$ siècle par des remontrances enflammées. Si cette simplification a fait florès jusque dans les années $1970^{2}$, les renouvellements historiographiques actuels, impulsés par les

1. MÉTHIVIER, Hubert, Le siècle de Louis XV, Paris, PUF, 1966, p. 64-65 et p. 115-116.

2. On peut citer parmi les tenants de cette historiographique largement antiparlementaire : GLASSON, Ernest Désiré, Le parlement de Paris, son rôle politique depuis le règne de Charles VII jusqu'à la Révolution, Paris, Hachette, 1901; MARION, Marcel, Le garde des sceaux Lamoignon et la réforme judiciaire de 1788, Paris, Hachette, 1905; GAXOTTE, Pierre, Le siècle de Louis XV, Paris, Fayard, 1933; Antoine, Michel, Louis XV, Paris, Fayard, 1989. 
Anglo-Saxons ${ }^{3}$ et poursuivis par les historiens français, ont montré la grande complexité des relations entre les parlements et la monarchie ${ }^{4}$.

Les historiens ont beaucoup débattu sur le rôle politique des parlements, mais peu se sont intéressés finalement à leur rôle majeur : l'activité judiciaire et réglementaire ${ }^{5}$. Cours souveraines agissant dans un ressort précis, les parlements étaient avant tout des cours de justice au sommet d'une pyramide, rendant justice aux particuliers se pourvoyant en appel, mais donnant aussi des arrêts sur propre mouvement du procureur général ou de la cour elle-même en cas de désordre dans la province. Étudier les parlements permet donc de donner un sens aux grands événements politiques de l'Ancien Régime, mais aussi de comprendre l'importance cruciale de cette institution dans l'organisation et l'équilibre d'une province, au même titre que l'intendant, arrivé cependant plus tardivement. Par ses fonctions, par sa correspondance quotidienne avec les juridictions subalternes, le parlement était un précieux informateur du pouvoir.

Le rôle du parlement dépassait donc largement ses prérogatives judiciaires, car il était pour le pouvoir une interface essentielle et commode et, s'il le désirait, un relais efficace. Or, pour apprécier ce rôle, une question mérite

3. HARDY, James D., Judicial politics in the Old Regime: the Parlement of Paris during the Regency, Bâton Rouge, Louisiana State University Press, 1967; DoyLE, William, "The Parlements of France and the breakdown of the Old Regime ", French Historical Studies, 1970, p. 415-458; CAMPBELl, Peter, Power and politics in Old Regime France: 1720-1745, Londres/New York, Routledge, 1996; Swann, Julian, Politics and the Parlement of Paris under Louis XV, 1754-1774, Cambridge, Cambridge University Press, 1995.

4. Pistes ouvertes en France en particulier par ÉGRET, Jean, Louis XV et l'opposition parlementaire, 1715-1774, Paris, Armand Colin, 1970. Chaline, Olivier, Godart de Belbeuf, le Parlement, le roi et les Normands, Luneray, Bertout, 1996; celui-ci a aussi donné l'impulsion à un certain nombre de rencontres rassemblant historiens français et anglais : Chaline, Olivier, SASSIER, Yves (dir.), Les Parlements et la vie de la cité, XVI $-X V I I I^{e}$ siècle, Rouen, Presses universitaires de Rouen, 2004, AuBERT, Gauthier, Chaline, Olivier (dir.), Les Parlements de Louis XIV. Opposition, coopération, autonomisation?, Rennes, PUR, 2011 ; Chaline, Olivier (dir.), Les parlements et les Lumières, Bordeaux, MSHA, 2012; LE MAO, Caroline (dir.), Hommes et gens du roi dans les parlements de la France moderne, Bordeaux, MSHA, 2011. À noter aussi les travaux impulsés par Alain J. Lemaître : LEMAîTRE, Alain-J., KAMMERER, Odile (dir.), Le pouvoir réglementaire : dimension doctrinale, pratiques et sources, actes du colloque de Mulhouse, Rennes, PUR, 2004 et LEMAîTRE, Alain-J. (dir.), Le monde parlementaire au XVIII siècle. L'invention d'un discours politique, Rennes, PUR, 2010. Il faut aussi signaler les actes d'un colloque tenu à Douai en 2011 : DAuchY, Serge, DEMARS-Sion, Véronique, Leuwers, Hervé, Michel, Sabrina (dir.), Les Parlementaires acteurs de la vie provinciale, Rennes, PUR, 2013. En amont, il faut noter l'importance des actes d'un colloque tenu dans les années 1990 à Toulouse, qui avait montré la richesse qu'il y avait à tirer de l'étude des parlements : Poumarède, Jacques, Thomas, Jack (dir.), Les Parlements de Province, pouvoirs, justice et société du $x^{e}$ au XVIII siècle, Toulouse, Framespa, 1996.

5. Ces questions ont surtout été traitées par les historiens du droit. Voir PAYEN, Philippe, Les arrêts de règlement du parlement de Paris au XVII 'e siècle. Dimension et doctrine, Paris, PUF, 1997,

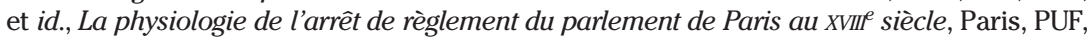
1999. Voir également sur ces mêmes arrêts : LEMONNIER-LESAGE, Virginie, Les arrêts de règlement du Parlement de Rouen, fin XVI'-XVII siècles, Paris, Panthéon-Assas, 1999 et plus récemment sur la "mécanique " du parlement de Flandre, DEMARS-Sion, Véronique, Michel, Sabrina, " Le Parlement de Flandre à travers ses archives ", Revue du Nord, t. 382, 2009, p. 679-918. 
d'être pleinement envisagée, et ne l'a guère été jusqu'ici : le fonctionnement interne de la cour, impliquant une connaissance précise de l'institution et la découverte de toute une mécanique, encore une fois largement ignorée au profit d'une histoire strictement politique. En effet, comment comprendre la collaboration ou l'opposition d'un parlement par ses remontrances, sans connaître le déroulement et les subtilités de l'assemblée des chambres ou de la rédaction des remontrances? Le parlement était une entité tripartite où les chambres, le parquet et le greffe avaient des fonctions précises et distinctes que les historiens commencent à cerner de plus en plus précisément ${ }^{6}$. Ils entrent ainsi dans l'intimité de la cour, à l'écoute des magistrats ou des gens du roi, ardents défenseurs de la loi, vigies de la jurisprudence provinciale et garants de l'ordre au sein du ressort. Dans ce cadre, il est clair que la démarche s'inscrit pleinement dans le cadre d'un certain " retour vers l'archive ${ }^{7}$ " favorisé par l'affaissement des grands cadres interprétatifs qui pouvaient finir par paraître plus importants que ce que les archives étaient susceptibles d'enseigner ${ }^{8}$. Une partie des chercheurs dont les textes sont rassemblés ici s'étaient d'ailleurs réunis en 2013 à Paris aux Archives nationales, sous la houlette de Françoise Hidelsheismer et de David Feutry, pour échanger sur ces aspects ${ }^{9}$. Le présent dossier s'intéresse donc à cette perspective en restreignant l'espace géographique à ce que l'on dénomme couramment l'Ouest de la France, où trois hautes cours de justice avaient autorité : le parlement de Paris, sur la Touraine, le Maine, l'Anjou et le Poitou; le parlement de Rennes (qui fut aussi parfois à Nantes et à Vannes) sur la Bretagne; le parlement de Rouen sur la Normandie. Malgré la richesse de ses archives, mais peut-être finalement à cause de cela, le parlement de Paris est le moins présent ici, et l'étude de son rayonnement sur les confins occidentaux de son ressort constitue probablement un horizon prometteur pour la recherche ${ }^{10}$. Reste que, par-delà les déséquilibres géographiques, les quelques leçons que l'on espère pouvoir tirer de la lecture des textes rassemblés peut avoir valeur pour d'autres endroits et entend constituer un appel à poursuivre dans la voie de la rénovation de l'histoire sociale, politique, institutionnelle de ces vieilles cours de justices vilipendées il y a tout juste cinquante ans par le grand historien Hubert Méthivier.

6. FEUTRY, David, Plumes de fer et robes de papier. Logiques institutionnelles et pratiques politiques du parlement de Paris au XVIII siècle, Paris, Fondation Varenne, 2013.

7. Voir le dossier " Fabrique des archives, fabrique de l'histoire ", Revue de synthèse, vol. 125, 2004, et pour le présent sujet, la contribution dans ce numéro de Françoise HiLDESHEIMER, "Exemplaire parlement... Le fonds du parlement de Paris aux Archives nationales ", p. 45-81.

8. AUBERT, Gauthier, Les Révoltes du Papier timbré (1675). Essai d'histoire événementielle, Rennes, PUR, 2014, p. 11-31.

9. Les parlements de France : logiques et pratiques documentaires (XVe-XVIII siècle), colloque organisé les 12 et 13 novembre 2012 à Paris par l'université Paris-Sorbonne, l'université Paris II Panthéon Assas et les Archives nationales.

10. Certaines affaires criminelles, étudiées par Reynald Abad, mentionnent les confins occidentaux du parlement de Paris, ABAD, Reynald, La grâce du roi. Les lettres de clémence de Grande Chancellerie au XVIII siècle, Paris, Presses de l'université Paris-Sorbonne, 2011. 